\title{
Uniqueness of galaxy groups in the structural hierarchy from its radio signature
}

\author{
Surajit Paul ${ }^{* \dagger}$ \\ Department of Physics, SP Pune University, Pune 411007, India \\ E-mail: surajit@physics.unipune.ac.in

\section{Prateek Gupta} \\ Department of Physics, SP Pune University, Pune 411007, India

\section{Reju Sam John} \\ Department of Physics, Pondicherry Engineering College, Puducherry, 605014, India

\section{Venkat Punjabi} \\ Department of Physics, SP Pune University, Pune 411007, India
}

\begin{abstract}
Galaxy groups are the intermediate structures in hierarchy of cosmic objects. Usually, galaxy clusters emerge out of mergers of groups of galaxies, but, no study confirms that groups are just the scaled down version of the clusters. In this work we have studied the thermal and non-thermal characteristics of galaxy groups from observational (SDSS catalogue) and theoretical (ENZO hydrodynamic simulations) point of view and compared them with the cluster properties. Our study shows that the mass scaling of both thermal and non-thermal properties of galaxy groups are deviating from cluster scale below $5 \times 10^{13} M_{\odot}$. A prominent break in the scaling laws at $5 \times 10^{13} M_{\odot}$ has been observed in computed radio emission as well. We have also estimated the cosmic magnetic field using turbulent dynamo model for these objects and found to be at a level of sub-micro Gauss. Possible radio synchrotron emission from groups have been calculated using both Diffusive Shock Acceleration and Turbulent Re-acceleration models. It is observed that the total emission from some of the groups are much higher than what is expected from existing scaling laws. Such groups must be away from virialization and are at a highly dynamic state. Finally, we have predicted the expected radio flux from these highly active groups for possible detection by future telescopes (e.g. SKA, Upgraded GMRT etc.).
\end{abstract}

EXTRA-RADSUR2015 (*)

20-23 October 2015

Bologna, Italy

(*) This conference has been organized with the support of the Ministry of Foreign Affairs and International Cooperation, Directorate General for the Country Promotion (Bilateral Grant Agreement ZA14GR02 - Mapping the Universe on the Pathway to SKA)

\footnotetext{
*Speaker.
} 


\section{Introduction}

In the structural hierarchy, galaxy groups are the intermediate objects between the small structures such as galaxies and the biggest rich galaxy clusters (Freeland \& Wilcots, 2011). But, unlike clusters that emerge at the filamentary nodes, groups usually form inside the dark matter filaments (Lietzen et al., 2012; Tempel et al., 2014; Vajgel et al., 2014). A simulated filamentary cosmological structure in Fig 1(Paul et al. 2016 in prep.) clearly reveals the abundance of groups inside the filaments that are arranged in a pattern resembling pearl necklace (Tempel et al., 2014). These groups and the Inter Galactic Material (IGM) eventually reach the galaxy clusters at the nodes of these filaments (Pimbblet, 2011; Perez et al., 2009; Moss, 2006). These groups while falling into the clusters, may attain a speed as high as few 1000s km/s (Rose et al., 2002).

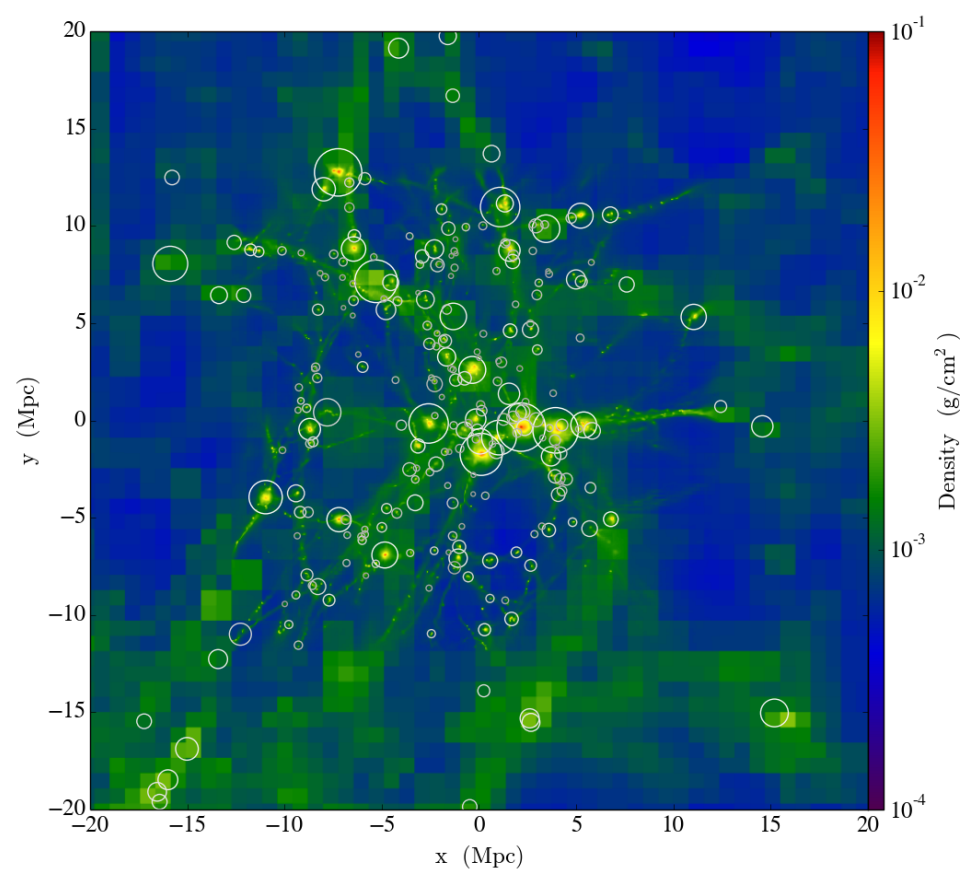

Figure 1: An area of $40 \mathrm{Mpc}^{2}$ hydro+DM simulated area with colour as density [S. Paul et al. 2016]. Galaxy groups (small white circles marked on filaments) and clusters (big circles at the nodes).

Our 3D simulation shows that the galaxy groups while falling in to the galaxy clusters, moves faster than the intergalactic material in the filaments. Because of this, groups may experience tremendous shear force and dynamical friction and will get stretched along the filaments. Such elongation along the filaments had also been observed in the groups of SDSS catalogues (Zhang et al., 2013). Elongation of groups induces collisions among the constituent galaxies (Struck, 2011) and will inject turbulence in the intra group medium. Moreover, due to their shallower gravitational potential (as they are inside the filaments), they are expected to be more strongly affected by processes such as mergers, feedback from super-massive black holes (SMBH), and galactic winds etc. (Lovisari et al., 2015). This will further ensure induction of instability in the IGM and filamentary material. Mach number dependent Cosmic ray content is also shown to be much larger in groups than in clusters and it differs by almost an order in magnitude (Jubelgas et al., 2008). Activity and fractional presence of AGN is much higher in groups and low mass clusters compared to massive clusters (Gilmour et al., 2007; Sivakoff et al., 2008). All these, strongly 
indicate that group medium is drastically different from the one in clusters and can be in a much turbulent state. Also, they are richer in pre-existing cosmic rays and possibly be producing higher amounts of non-thermal energy than the usual expected level predicted by extrapolation from the cluster properties. In-fact, a clear deviation of scaling laws has been reported for groups from clusters for $L_{\mathrm{x}}-\mathrm{T}$ and velocity dispersion (i.e. $\sigma$ ) relations by Bharadwaj et al. (2015).

Though, in last few years, some researchers worked on the thermal properties of groups (Bharadwaj et al., 2015; Vajgel et al., 2014; Pratt et al., 2009), almost no study has been done on their non-thermal properties. Since, non-thermal physics is very much dependent on transient activities such as mergers, shocks and turbulence etc., it can reveal much more information about the dynamical and evolutionary physics of large scale structures (for review Dolag et al. (2008); Brüggen et al. (2012)). So, a thorough study of non-thermal physics in galaxy groups is well in demand. Non thermal quantities like cosmic magnetism and radio synchrotron emissions are very much dependent on turbulence in the system. Amplification of magnetic field to the level that we observe can be modelled with good accuracy by turbulent dynamo (Subramanian et al., 2006) and the acceleration of particles that produce radio halo emission can be modelled by Diffusive shock acceleration and turbulent re-acceleration(Brunetti \& Lazarian, 2007). So, a well studied turbulence in galaxy group systems will surely help us in studying the dynamics of structure formation and even will help to reveal the Worm Hot Intergalactic Medium (WHIM) that is supposed to be residing in the filaments.

\section{Radio emission from simulated galaxy groups}

Very poor knowledge on properties of galaxy groups prompted us to theoretically model them using cosmological simulations. To create our sample of galaxy groups, basic simulations were performed with the adaptive mesh refinement (AMR), grid-based hybrid (N-body plus hydrodynamical) code Enzo v. 2.1 (O'Shea et al., 2004). With introduction of 2 nested child grid and 4 levels of AMR at the central $32 \mathrm{Mpc}^{3}$ volume we have achieved a resolution of $\sim 30 \mathrm{kpc}$ at the highest level. As additional physics, radiative cooling is included (Sarazin \& White, 1987) for a fully ionized gas with metallicity of 0.5 solar and a star formation feedback scheme has been applied (Cen \& Ostriker, 1992).

We have chosen $\sim 500$ groups with masses from $5 \times 10^{12} M_{\odot}$ to cluster mass $\sim 10^{15} M_{\odot}$ from 10 simulations of $128 \mathrm{Mpc}^{3}$ volume. Our mass resolution at the smallest child grid is $<10^{9} M_{\odot}$ providing enough mass resolution for the groups. Also, with $\sim 30 \mathrm{kpc}$ spatial resolution, systems above $5 \times 10^{12} M_{\odot}$ with mostly having virial radii above $300 \mathrm{kpc}$ get adequately resolved in space.

In each galaxy group, we have computed the local velocity dispersion by filtering out the bulk motion of baryons in the core of the groups. A velocity dispersion of 100 to $300 \mathrm{~km} / \mathrm{s}$ has been observed from our simulated groups. Clusters, obviously show much larger values. Strikingly, some groups with very low mass show much higher local velocity dispersion, almost at the level of clusters (Fig. 2, panel 1).

The magnetic field of $\mu \mathrm{G}$ that we usually observe in the Inter Cluster Medium can be achieved though turbulent dynamo. This mechanism can boost the level of ICM seed magnetisation of $10^{-21}$ Gauss to present value of $\mu \mathrm{G}$. Such kind of amplification of magnetic field certainly needs a very high degree of turbulence in the medium. A saturation of magnetisation can be achieved in a fully turbulent medium. An equipartition can be considered between magnetic energy density $\frac{B^{2}}{8 \pi}$ and the kinetic energy density $\rho \varepsilon_{\text {turb }}$ in this condition. Saturated magnetic field is then estimated from the hydrodynamic parameters by the given relation $\frac{B_{\text {sat }}^{2}}{8 \pi} \propto \rho \varepsilon_{\text {turb }}$ i.e. $B_{\text {sat }}=\sqrt{C_{E} \cdot 4 \pi \cdot \rho v_{\text {rms }}^{2}}$ (Subramanian, et al., 1998; Iapichino et al., 2012), where, $v_{\text {rms }}$ is the local velocity dispersion. 

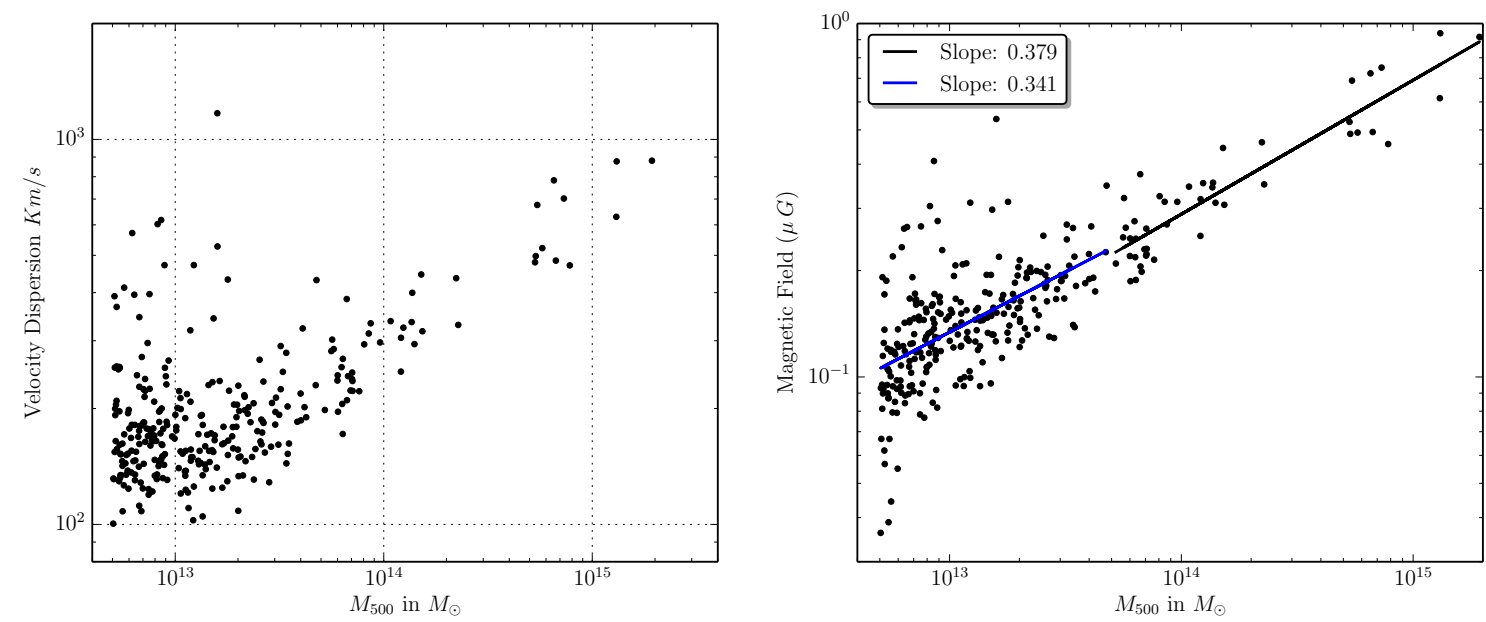

Figure 2: Panel 1: Velocity dispersion of baryons vs the mass of the groups from simulations. Panel 2: Computed magnetic field plotted $v$ s the mass of the simulated groups.

The constant of proportionality $C_{E}$ is at most 0.05 (Miniati \& Beresnyak, 2015). Implementing this model, we find a sub $\mu \mathrm{G}$ magnetic field in our simulated sample of groups (Fig. 2, panel 2). The slopes of the smaller mass systems $\left(<5 \times 10^{13} M_{\odot}\right)$ are a little flatter. But the important observation is, there are groups with very high magnetic field and are much away from the cluster scaling.

The electron energy spectrum that determines the actual synchrotron power can be computed from either diffusive shock acceleration (DSA or Fermi I) method (Drury, 1983) or by turbulent re-acceleration (TRA or Fermi II) (Brunetti \& Lazarian, 2007). The non-thermal particles are approximated by a power law energy distribution $N(E) d E \propto E^{-\delta} d E$. Where, $\delta$ is the spectral index of electron energy with value 2 and more steeper. With TRA, the electron energy power law takes up the form $\left(\frac{d N_{e}}{d E_{e} d V}\right)_{\mathrm{inj}}=\frac{3 P_{A} c}{4 S\left(E_{\max }\right)^{1 / 2}} E_{e}^{-\delta}$. A simple assumption would be to consider a fully developed Kolmogorov type turbulence that makes the $\delta$ to be $\frac{5}{2}$ (Fang $\&$ Linden, 2015). Radio luminosity computed using this models at $1.4 \mathrm{GHz}$ revealed that the radio emission is much greater in the groups than what is expected from the observed cluster scaling. Scaling of radio emission with mass breaks away from cluster correlation at $\sim<5 \times 10^{13} M_{\odot}$ (Fig. 3, panel 1).
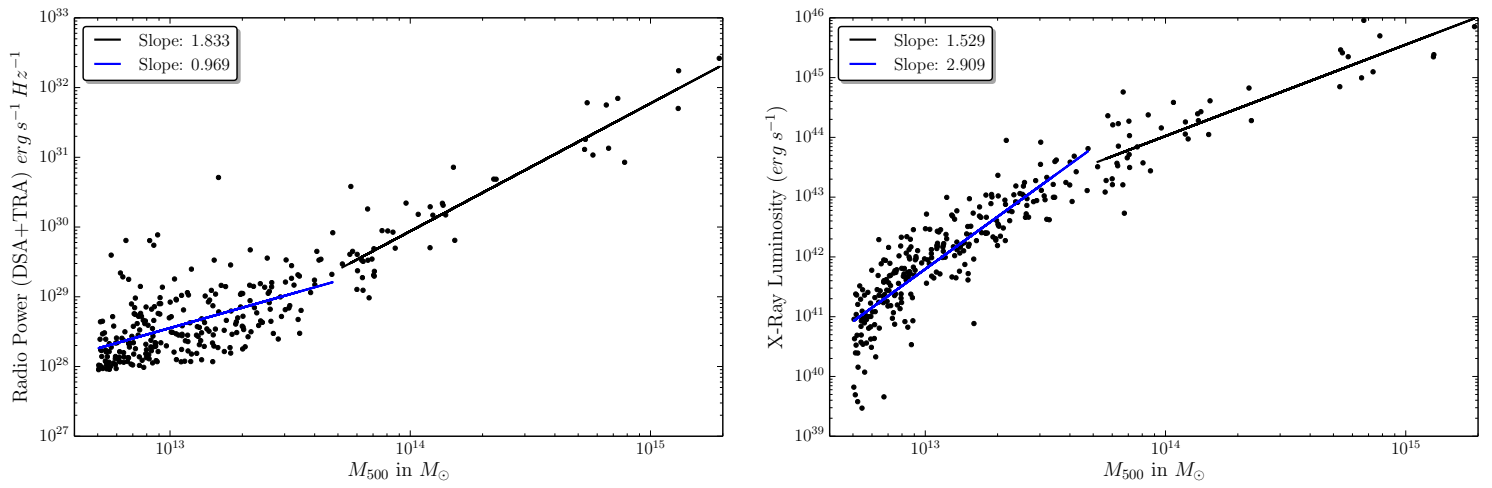

Figure 3: Panel 1: Modelled radio power (DSA \& TRA) from simulated groups and clusters. Panel 2: Computed X-ray luminosity using CLOUDY code from the simulated galaxy groups. 
Deviation from cluster scaling in non-thermal radio emission for the groups prompted us to verify its thermal property. We have computed the temperature weighted X-ray emission from these groups utilizing the Cloudy (Ferland et al., 2013) code. Calculations were done for the photon energy ranging from $0.1 \mathrm{keV}$ to $12.0 \mathrm{keV}$. As expected, a deviation in scaling was found at a similar point i.e. $\sim 5 \times 10^{13} M_{\odot}$ for x-ray emission as well from the simulated groups (Fig. 3, panel 2). Here, the change in the slope is just opposite, going steeper in groups unlike in non-thermal cases.

\section{Expected radio emission from SDSS groups}

For observational studies, we have chosen the galaxy group catalogue of Tempel et al. (2014) as our primary data source. This catalogue has been prepared using the Sloan Digital Sky Survey (SDSS, DR-10) data. On the tabulated galaxy data, authors applied Friends of Friends algorithm (FoF), and identified a total of 82,458 galaxy groups by defining groups as an object having at least 2 galaxies and choosing suitable linking length (see Tempel et al. (2014) for details). For our study, we have initially chosen groups with at least 10 galaxy candidates and having total mass greater than $10^{12} M_{\odot}$. So, in our initial sample we got 2903 groups and their masses are in the range of $1.5 \times 10^{12}-2 \times 10^{15} \mathrm{M}_{\odot}$.

For these groups, we have computed the magnetic fields using the method described in Section 2. We found the magnetic field to be varying in the range of sub $\mu G$ to a few $\mu G$ for the biggest objects and follows the same pattern as it is observed from the simulated groups. Similarly, we have computed the radio emission, both from the DSA and turbulent re-acceleration electrons from the SDSS groups at $1.4 \mathrm{GHz}$ (Fig. 4, panel 1). Again, the same pattern of a break in the scaling at $\sim 5 \times 10^{13} M_{\odot}$ has been observed. Low mass objects i.e. galaxy groups show a flatter variation of radio emission. Similar to the simulated objects, we have computed the possible X-ray emission from the SDSS groups as well (Fig. 4, panel 2). Though the slopes are a little different, but the qualitative behaviour remained the same.
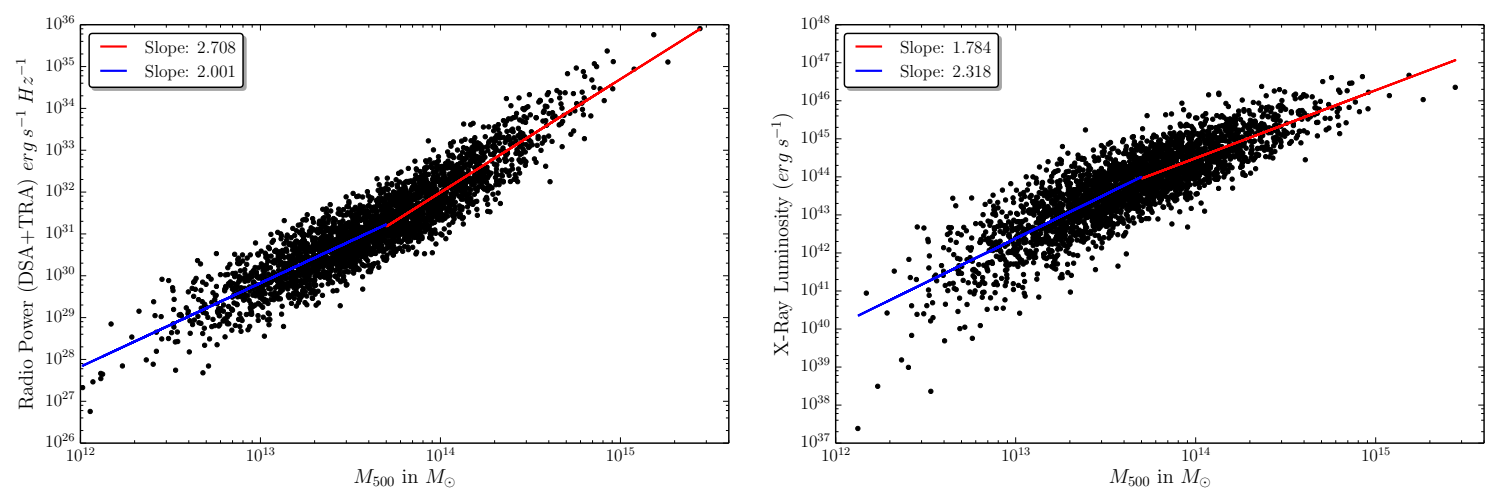

Figure 4: Panel 1: Modelled radio power (DSA \& TRA) from SDSS groups and clusters. Panel 2: Computed $X$-ray luminosity using CLOUDY code from the SDSS groups.

Such deviation in the scaling laws, indicates higher turbulent energy and higher level of radio emission, especially for some of the groups (See Fig. 3). This gives us hope that if we can methodically select these high radio emitting objects, it will be possible to observe non-thermal emission from very small structures like galaxy groups. For this purpose, we have chosen the deviation from virialization of a system as the indication of high activity. The virialization is quantified via the ratio of potential energy (P.E.) $=\frac{3}{5} \frac{G M}{R}$ and twice of the kinetic energy (K.E.) $=\frac{3}{2} \frac{K_{B} T}{m_{p}}$. So, a highly active system will have virial ratio $<<1$. 
We have plotted a frequency histogram of the virial ratio and fitted it with a Lorentzian curve. The distribution peaks at 0.99 with a width of 0.49 (i.e. $\sigma$ ), which is very near to the virialization condition of 1 (see Fig 5, panel 1). Roughly, we can assume this peak as the virialization and then categorize the whole sample on the basis of how far an object is from virialization. Since, $\left(\frac{\text { P.E. }}{2 \mathrm{~K} . \mathrm{E} .}\right)$ $<1$ indicates much higher kinetic energy or turbulence in the medium, the groups that are one $\sigma$ below the peak (i.e. ratio 0.5 ), can be taken as highly active groups. We found 158 such objects among the total 2903 objects in our sample (i.e. 5\%). It has been noticed that, most of the active systems are actually the low mass systems with mass below $10^{14} M_{\odot}$. This vindicates our claim that groups are mostly away from virialization and have a high degree of turbulence in their medium.
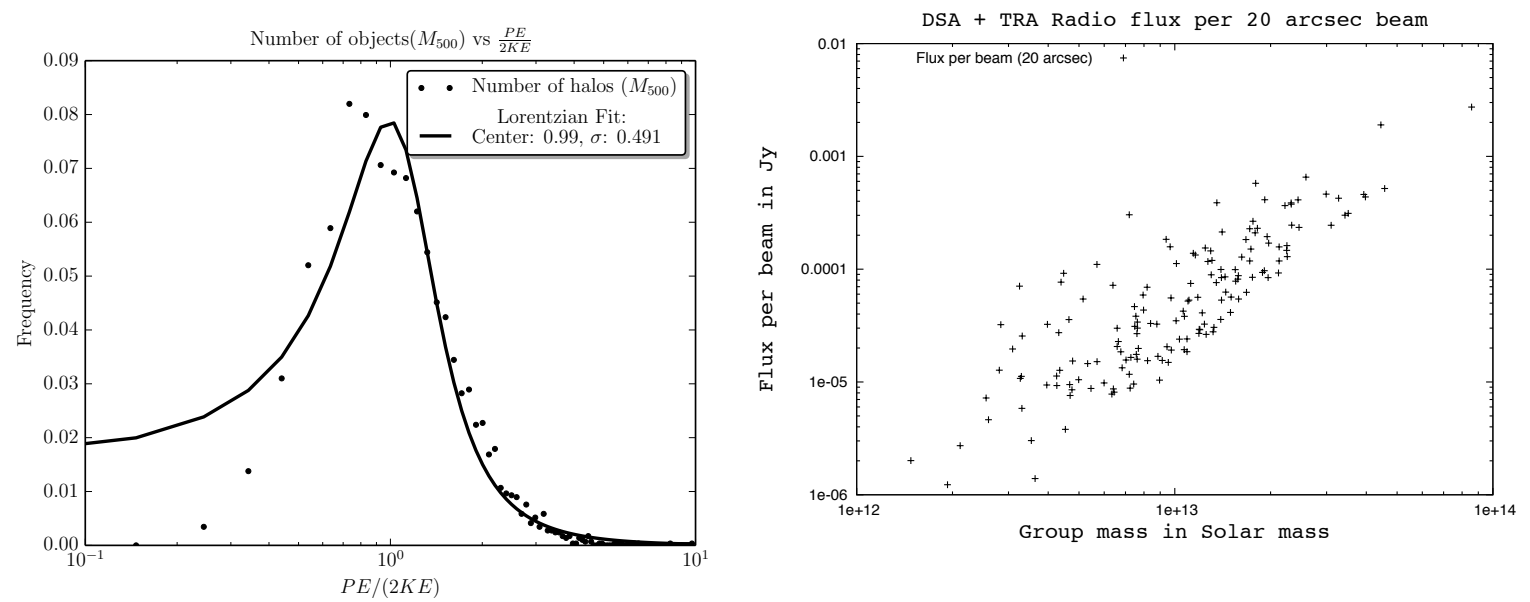

Figure 5: Panel 1: Frequency histogram of energy ratio $\left(\frac{\text { P.E. }}{2 \text { K.E. }}\right)$ on a normalized scale. Panel 2: Total radio flux (DSA+TRA) per beam (20") from most active groups plotted $v$ s the group mass.

Applying our theoretical model of radio emission to these highly active SDSS groups, we have estimated the $1.4 \mathrm{GHz}$ flux per beam $\left(20^{\prime \prime}\right)$. The computed flux varies in the range of sub $\mu \mathrm{Jy}$ to few 10s of $\mu \mathrm{Jy}$ per beam (see Figure 5, panel 2). Many of these objects are showing promising level of fluxes that are even within the range of few existing upgraded radio telescopes.

\section{Discussion and conclusions}

Galaxy groups being placed intermediate to the field galaxies and the clusters of galaxies, are a very important part of cosmological structures. We modelled their energetics using cosmological simulations and also implemented them on observed SDSS galaxy groups to compute different parameters. Our study establishes uniqueness of the groups in the structural hierarchy.

- We found that the velocity dispersion in low mass structures are higher than expected.

- Saturated magnetic fields are estimated for our simulated and SDSS samples and are found to be in the range of sub $\mu \mathrm{G}$. Strikingly some of them show higher values, almost at a level of big galaxy clusters.

- Both thermal and non-thermal emissions i.e. X-ray and Radio emission power show a sharp break in scaling at $\sim 5 \times 10^{13} M_{\odot}$. This indicates, structures with mass below $5 \times 10^{13} M_{\odot}$ are a different set of structures with different physical conditions or energetics. This helped us 
in defining a new category of objects i.e. Galaxy groups. So, groups are not the scaled down version of galaxy clusters and needs to be studied very deeply to understand the underlying physics that makes them different from the clusters.

- The overall radio emission plot (Fig. 4, panel 1) shows some of the groups having very high radio powers. We defined them as highly active groups. We then relate them to the low virial ratio systems as described in the Section 3. Finally, we have chosen a list of highly active groups from the SDSS-group catalogue and computed the expected radio flux from those objects. Expected fluxes are in the range of few $\mu \mathrm{Jy}$ and can possibly be detected with the upgraded or future generation radio telescope arrays like UGMRT, SKA, LOFAR etc.

\section{Acknowledgement}

We are very much thankful to The Inter-University Centre for Astronomy and Astrophysics (IUCAA) for providing the Computing facility. Computations described in this work were performed using the Enzo code developed by the Laboratory for Computational Astrophysics at the University of California in San Diego (http://lca.ucsd.edu). We are also thankful to DST INSPIRE Faculty scheme for funding this project. SP also likes to thank SERB, Govt. of India for providing the travel fund to present this work.

\section{References}

Bharadwaj, V., Reiprich, T. H., Lovisari, L., \& Eckmiller, H. J. 2015, AAP, 573, A75

Brüggen, M., Bykov, A., Ryu, D., Rẗtgering, H. 2012, SSR, 166, 187

Brunetti, G., \& Lazarian, A. 2007, MNRAS, 378, 245

Cen, R., \& Ostriker, J. P. 1992, ApJl, 399, L113

Dolag, K., Bykov, A. M., \& Diaferio, A. 2008, SSR, 134, 311

Drury, L. O. 1983, Reports on Progress in Physics, 46, 973

Fang, K., \& Linden, T. 2015, arXiv:1506.05807

Ferland, G. J., Porter, R. L., van Hoof, P. A. M., et al. 2013, RMXAA, 49, 137

Freeland, E., \& Wilcots, E. 2011, ApJ, 738, 145

Gilmour, R., Gray, M. E., Almaini, O., et al. 2007, MNRAS, 380, 1467

Iapichino L., \& Brüggen M., 2012, MNRAS, 423, 2781

Jubelgas, M., Springel, V., Enßlin, T., \& Pfrommer, C. 2008, AAP 481, 33

Lietzen, H., Tempel, E., Heinämäki, P., et al. 2012, AAP, 545, A104

Lovisari, L., Reiprich, T. H., \& Schellenberger, G. 2015, AAP, 573, A118

Miniati, F., \& Beresnyak, A. 2015, Nature, 523, 59

Moss, C. 2006, MNRAS, 373, 167

O'Shea, B. W., Bryan, G., Bordner, J., et al. 2004, arXiv:astro-ph/0403044

Paul, S., Iapichino, L., Miniati, F., Bagchi, J., \& Mannheim, K. 2011, ApJ, 726, 17

Perez, J., Tissera, P., Padilla, N., Alonso, M. S., \& Lambas, D. G. 2009, MNRAS, 399, 1157 
Pimbblet, K. A. 2011,MNRAS , 411, 2637

Pratt, G. W., Croston, J. H., Arnaud, M., Böhringer, H. 2009, AAP, 498, 361

Rose, J. A., Gaba, A. E., Christiansen, W. A., et al. 2002, AJ, 123, 1216

Sarazin, C. L., \& White, R. E., III 1987, ApJ, 320, 32

Sivakoff, G. R., Martini, P., Zabludoff, A. I., Kelson, D. D., \& Mulchaey, J. S. 2008, ApJ, 682, 803

Struck, C. 2011, Galaxy Collisions by Curtis Struck. New York: Springer, 2011. ISBN:978-0-38785370-3,

Subramanian et al., 1998, MNRAS, 294, 718

Subramanian K., et al.2006, MNRAS, 366, 1437

Tempel, E., Kipper, R., Saar, E., et al. 2014, AAP, 572, A8

Tempel, E., Tamm, A., Gramann, M., et al. 2014, AAP, 566, A1

Vajgel, B., Jones, C., Lopes, P. A. A., et al. 2014, arXiv:1409.6822

Zhang, Y., Yang, X., Wang, H., et al. 2013, ApJ, 779, 160 